\title{
Investigating Ferroelectric Domain and Domain Wall Dynamics at Atomic Resolution by TEM/STEM in situ Heating and Biasing
}

Michele Conroy $^{1 *}$, Kalani Moore ${ }^{1}$, Eoghan O'Connell ${ }^{1}$, Eileen Courtney ${ }^{1}$, Alan Harvey ${ }^{1}$, Charlotte Cochard $^{2}$, Joseph Guy ${ }^{2}$, Raymond McQuaid ${ }^{2}$, Lewys Jones ${ }^{3}$, Clive Downing ${ }^{3}$, Roger Whatmore ${ }^{4}$ and Marty Gregg ${ }^{2}$, Ursel Bangert ${ }^{1}$

1. TEMUL, Department of Physics, School of Natural Sciences \& Bernal Institute, University of Limerick, Limerick, Ireland.

2. Department of Mathematics and Physics, Queen’s University, University Rd., Belfast, UK.

3. Advanced Microscopy Laboratory, Trinity College Dublin, Dublin, Ireland.

4. Department of Materials, Imperial College London, Exhibition Road, London, UK.

* Corresponding author: Michele.conroy@ul.ie

Conducting domain walls (DWs) in ferroelectrics is an emerging research focus in nano-electronics [1,2]. Previously overlooked, these walls have recently been reported to possess diverse functional characteristics that are completely different from the domains that they delineate [3-5]. They can have their own distinct chemistry and magnetic behavior [6], and in turn represent a completely new sheet phase. The characteristics of these confined regions are believed to have the same exotic functional behaviours as seen in 2D materials such as graphene, opening up a plethora of possible electronic applications. In addition, the walls have the unique property of being 'agile'; they can be created or destroyed and even be controllably moved by an external field. However, this is an area of research at its very early stages, with a great deal of the fundamental physics still unknown. Since the region of interest (the domain wall) is atomically thin and dynamic, it is essential for the physical characterization to be at this scale and be time-resolved.

In this presentation we will focus on the conducting (DWs) of the improper ferroelectric copper chlorine boracite $\left(\mathrm{Cu}_{3} \mathrm{~B}_{7} \mathrm{O}_{13} \mathrm{Cl}\right)$ in single crystal form. The electron microscopy study was conducted at various spatial resolution to compliment the previous DW dynamics by light microscopy [1]. The DW movement was initially studied by scanning electron microscopy (SEM) of the bulk crystal before scanning transmission electron microscopy (STEM) lamella focused ion beam (FIB) preparation. SEM imaging gave us an overview of the DW pattern at the micron/nanometer scale from the top surface of the crystal before moving to STEM spatial resolution. The DW and phase difference of the neighboring domains were imaged by STEM high angle annual dark field and annual bright field. By quantifying the atomic displacement using Atomap [7] we can directly measure the localized polarization at the wall. To ensure the correct atoms and thus correct charge was assigned to the imaged atoms, STEM HAADF and annular bright field simulations were also carried out to allow comparison to the experimental data. By simulating the strain on the unit cell in different directions, using Mathematica, we can confirm the link between strain and resulting polarization.

We then investigated the injection and controlled movement of the DWs by in situ heating and biasing (DENS lightning holder). Low dose transmission electron microscopy (TEM) imaging allowed us to avoid the movement of the walls due to the STEM probe and focus on the movement due to the in situ heating and biasing. We conducted a detailed phase change study of the DW injection by in situ heating, revealing the complex but repeatable nature that produces the charged and uncharged DWs. The in situ 
biasing experiments revealed there is a direct relationship between the charged and uncharged DW movement.

Our results show in situ electron microscopy provide a new platform for understanding the fundamentals of ferroelectric material domain phase change and thus DW injection and movement. These new insights needed the resolution allowed by STEM/TEM due to the 2D nature of the walls [8].

\section{References:}

[1] R McQuaid et al., Nature Communications 8 (2017).

[2] J Jiang et al., Nature Materials 17 (17) (2017), p. 49.

[3] LJ McGilly et al., Nature Nanotechnology 10 (2015), p. 145.

[4] J Seidel et al., J. Phys. Chem. Lett 19 (2012), p. 2905.

[5] G Catalan et al., Domain Wall Nanoelectronics. Rev. Mod. Phys 84 (2012), p. 119.

[6] S Farokhipoor et al., Nature 515 (2014), p. 379.

[7] M Nord et al., Adv Struct Chem Imaging 3 (2017) p. 9.

[8] The authors acknowledge funding from SFI US-Ireland fund.
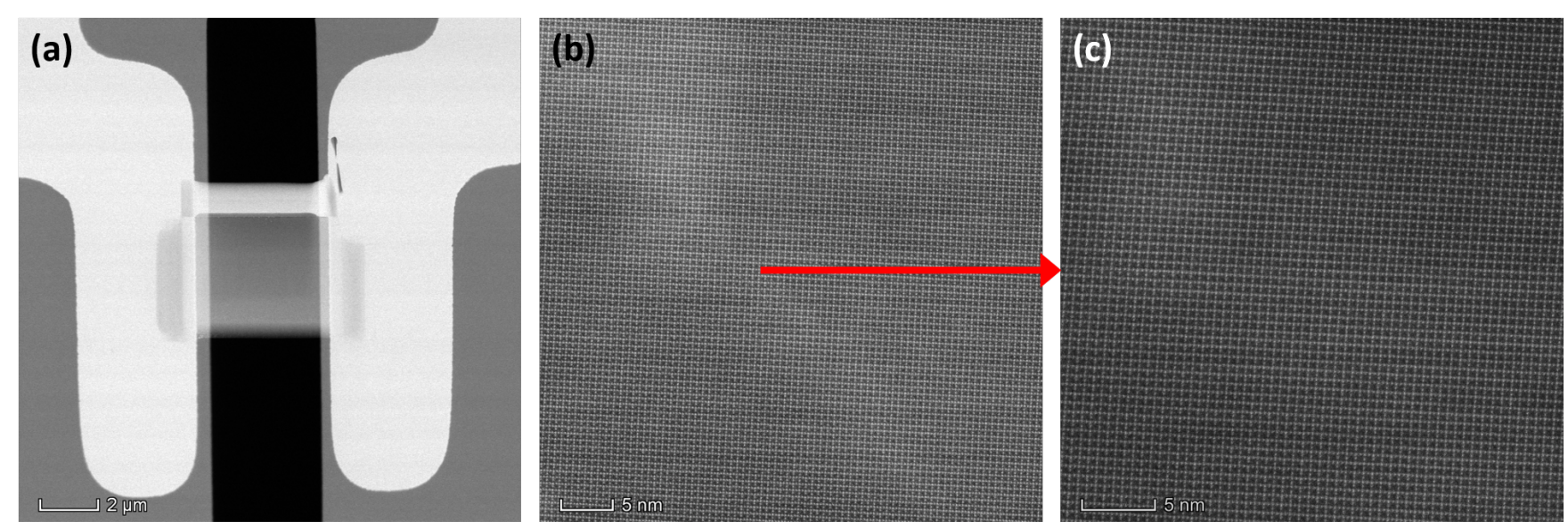

Figure 1. STEM HAADF imaging of (a) low resolution FIB lamella welded on to heating and biasing in situ TEM chip, (b), (c) atomic resolution images showing the domain wall. 